\title{
Protective effects of adipose-derived multipotent mesenchymal stromal cells of mice on periventricular leukomalacia model in vitro
}

\author{
Tsupykov O. M. ${ }^{1,2}$, Lushnikova I. V. ${ }^{1}$, Ustymenko A. M. ${ }^{2}$, Kyryk V. M. ${ }^{2}$, Nikandrova Y. A. ${ }^{1}$, Patseva M. A. ${ }^{1}$, \\ Yatsenko K. V. ${ }^{1}$, Butenko G. M. ${ }^{2}$, Skibo G. G. ${ }^{1,2}$ \\ ${ }^{1}$ Bogomoletz Institute of Physiology NAS of Ukraine, Kyiv, Ukraine \\ ${ }^{2}$ State Institute of Genetic and Regenerative Medicine NAMS of Ukraine, Kyiv, Ukraine \\ e-mail: tsupykov@gmail.com
}

\section{ABSTRACT}

Periventricular leukomalacia (PVL) is a form of white matter lesions of the brain that results from hypoxic-ischemic injury and/or inflammation of nervous tissue, and is one of the causes of cerebral palsy. On PVL models in vivo, we have demonstrated neuroprotective effect of transplantation of adipose-derived multipotent mesenchymal stromal cells (MMSCs). However, the mechanisms, which realize neuroprotective effect of transplanted MMSCs, remain unexplored.

THE AIM was to assess the influence of adipose-derived MMSCs on cultured mouse brain slices at their contact co-culturing on PVL models in vitro.

METHODS. Periventricular leukomalacia in vitro was modelled by a 30-minute oxygen-glucose deprivation (OGD) of mouse brain slices, followed by the addition of $100 \mathrm{ng} / \mathrm{ml}$ LPS in culture medium. For co-cultivation we used adipose-derived MMSCs obtained from mice FVB$\mathrm{Cg}-\mathrm{Tg}$ (GFPU) $5 \mathrm{Nagy} / \mathrm{J}$, transgenic for green fluorescent protein (GFP). The viability of cultured sections cells was evaluated by analysing the level of lactate dehydrogenase (LDH) in the culture medium. Probable MMSCs differentiation into neurons and glial cells was studied using immunohistochemical staining of slices using specific antibodies to neurons and oligodendrocytes (NeuN and Oligodendrocytes, respectively).

RESULTS. Modelling of PVL in vitro on organotypic culture of brain slices led to a significant increase in level of cytosolic enzyme LDH in the culture medium. Co-cultivation of slices with MMSCs at PVL reduced the amount of this enzyme. Furthermore, it is shown that under conditions of PVL in vitro, MMSCs are able to differentiate into cells of nervous tissue.

CONCLUSIONS. Adipose-derived MMSCs have protective effect when they are co-cultivated with the mice brain slices on PVL model in vitro.

KEYWORDS: periventricular leukomalacia; lipopolysaccharide; brain organotypic tissue culture; multipotent mesenchymal stromal cells

Periventricular leukomalacia (PVL) is a form of the brain white matter lesions, characterized by oligodendrocytes death - cells responsible for myelination of axons and astro- and microgliosis in the periventricular area of the brain $[3,9]$. Damage to the myelination of axons results in disorders of brain signaling that manifests itself in the deterioration of motor control, delayed physical, intellectual and emotional development of children $[3,6,21]$. Because of underdevelopment or brain damage in the prenatal, intrapartum and early postnatal periods may occur a group of syndromes, which are combined by a term «cerebral palsy» (CP).
There are many possible reasons for PVL; the main ones are hypoxicischemic brain damage, autoimmune mechanisms in the motherfetus system, intrauterine infections, especially viral, etc. Recently, an important role in the PVL pathogenesis is given to neuroimmune conflict in the mother-fetus system that leads to violation of both central nervous system and the immune system of the fetus $[11,12,24]$.

Current pharmacological therapy of perinatal pathology of CNS is imperfect and can disrupt the complex relationship compensatory-adaptive processes in the body of the child, which often leads to complications that 
prevent the continuation of drug therapy [23]. Rehabilitation measures in such cases should be held in the first months of life, when the largest renewable effect can be expected.

Cell therapy with stem cells is a promising approach to the treatment of many CNS diseases, including PVL $[8,14,18]$. Despite numerous studies in this area, the question remains open not only about supporting roles of transplant cells in regeneration, but also the possibility of trans-differentiation of other non-neural stem cells such as multipotent mesenchymal stromal cells (MMSCs) into neurons or glial cells $[7,19]$. MMSCs have a tropism for the damage area and can affect the progress of inflammation and repair it. [1] They provide the recipient immune system tolerance to allogeneic cells, actually themselves. The use of autologous MMSCs would solve the problem of immunological compatibility of transplant material and its testing for infection, and avoid ethical and legal restrictions on fetal donor material [4]. It was shown that in the transplantation of adipose-derived MMSCs on a model of hemorrhagic stroke in rats the neurological deficits are weakened, brain atrophy is reduced, myocardial ischemic area is significantly reduced and a number of small vessels is growing [10]. In previous work on periventricular leukomalacia model in vivo, we also demonstrated the neuroprotective effect of transplantation of adipose-derived MMSCs [20]. However, the disclosure of mechanisms by which transplanted MMSCs promote cell survival and functional recovery of animals requires further research.

In this paper, we studied the effect of adipose-derived MMSCs on the cultured mouse brain slices at their contact co-culturing on the PVL models in vitro.

\section{MATERIALS AND METHODS}

All animal experiments were performed in compliance with international principles of the European Convention for the Protection of vertebrate animals used for experimental and other scientific purposes (European convention, Strasburg, 1986), Article 26 of the Law of Ukraine «On protection of animals from cruelty» (№ 3447-IV , 21.02.2006) and all norms of bioethics and biosafety.

In our study, we used mice FVB «wild» type and FVB-C$\mathrm{Tg}$ (GFPU)5Nagy/J, transgenic for green fluorescent protein (GFP), which were kept under standard conditions at the vivarium of SI IHRM with free access to water and food.

Organotypic culture of mice brain slices. Organotypic culture of the brain slices was obtained from 7-day-old FVB mice [21]. After a quick decapitation under ether anesthesia, the brain was isolated, divided into two parts by the median line; and we made $350 \mu \mathrm{m}$ thick cuts with automatic chopper (Mclllwain, England). The sections were cultured on porous semipermeable nitrocellulose membranes Millicell-CM (Millipore, USA), placed in a $\mathrm{CO}_{2}$ incubator at the border of gas (a mixture of air with $5 \% \mathrm{CO}_{2}$ ) and liquid medium ( $\mathrm{pH}=7.2$ ) containing $50 \% \mathrm{MEM}, 25 \%$ balanced salt solution Hanks, $25 \%$ inactivated horse serum, $10 \mathrm{mM}$ Tris, $2 \mathrm{mM} \mathrm{NaHCO}{ }_{3}, 12.5 \mathrm{mM}$ HEPES, $15 \mathrm{mM}$ glucose, $100 \mathrm{U} / \mathrm{ml}$ penicillin, $100 \mu \mathrm{g} / \mathrm{ml}$ streptomycin (all - Sigma, USA) in 6-well plates at $35^{\circ} \mathrm{C}$. The culture medium was changed on the second day of incubation and then twice a week.

Obtaining of multipotent mesenchymal stromal cells from adipose tissue. Adipose tissue of FVB-Cg-Tg(GFPU) $5 \mathrm{Nagy} / \mathrm{J}$ mice, transgenic for the GFP gene, was used to obtain MMSCs by the previously described method [20]. Fragments of crushed subcutaneous adipose tissue from the groin area were fermented in $0.1 \%$ collagenase $1 \mathrm{~A}$ solution (Sigma, USA) for $90 \mathrm{~min}$ at $37^{\circ} \mathrm{C}$ in a shaker at $100 \mathrm{rpm}$. The resulting suspension was washed in culture medium DMEM (Sigma, USA) by centrifugation at 400x g. We collected supernatant, which contained mature adipocytes, resuspended the precipitate in DMEM medium containing $15 \%$ fetal bovine serum (FBS) and passed through a cell filter with pore diameter of
100 microns. The cells were seeded in $25 \mathrm{~cm}^{2}$ culture flasks at a density of $5 \cdot 10^{5}$ cells $/ \mathrm{cm}^{2}$ and cultured in complete nutrient medium DMEM-HG (Sigma, USA) containing $10 \%$ FBS (HyClon, USA), antibiotics (penicillin $100 \mathrm{U} / \mathrm{ml}$, streptomycin $100 \mathrm{mg} / \mathrm{ml}$, (Sigma-Aldrich, USA), 1:100 nonessential amino acids (Sigma-Aldrich, USA) in a $\mathrm{CO}_{2}$ incubator under conditions of moist air with $5 \% \mathrm{CO}_{2}$ at $+37^{\circ} \mathrm{C}$. Passaging of the cultures was carried out at reaching $80 \%$ confluency of a monolayer using $0.25 \%$ trypsin solution (Sigma, USA).

Phenotyping of cells from the resulting cultures was performed on the $2^{\text {nd }}$ passage by flow cytometry using a rat anti-mouse IgG1 monoclonal antibody against the markers CD44-PE, CD73-PE, CD90-PE, CD34-APC, CD45-PE and CD117-Pe-Cy7 ( all - Becton Dickinson, USA) in the work concentration of $0.5 \mu \mathrm{g} / \mathrm{ml}$. The percentage of dead and viable MMSCs was measured in terms of penetration of 7-amino actinomycin D (Becton Dickinson, USA) into cells with damaged membrane. Phenotyping of adipose-derived MMSCs culture confirmed a high level of expression of markers CD44, CD73, CD90 $(84-96 \%)$, while the relative content of cells expressing hematopoietic markers CD34, CD45 and CD117 was less than $2 \%$.

To confirm multipotent properties of the MMSC culture of the $2^{\text {nd }}$ passage, we conducted directed differentiation for 21 days in osteogenic and adipogenic directions. Complete culture medium for osteogenic differentiation medium consisted of DMEM-F12 (Sigma, USA) supplemented with $10 \% \mathrm{FBS}$, and contained L-ascorbic acid 2-phosphate $(0.05 \mathrm{mM})$, dexamethasone $(100 \mathrm{nM})$ and $\beta$-glycerophosphate $(10 \mathrm{mM})$. Complete culture medium for adipogenic differentiation medium consisted of DMEM-F12 (Sigma, USA) with the addition of $1 \mu \mathrm{M}$ dexamethasone, $200 \mu \mathrm{M}$ indomethacin, $500 \mu \mathrm{M}$ isobutyl methylxanthine and $5 \mu \mathrm{g} / \mathrm{ml}$ insulin (all - Sigma, USA).

The postponement of calcium in the extracellular matrix of cultured cells was detected by staining preparations fixed in $4 \%$ formaldehyde solution with Alizarin Red S. The production of alkaline phosphatase was confirmed by staining with BCIP/NBT (Sigma, USA). Visualization of lipid granules in the cytoplasm of cells was performed by staining with Oil Red 0 (Sigma, USA).

According to morphological characteristics, immunophenotype and potential for directed differentiation obtained adipose cell cultures, which were used in further experiments complied with the minimal criteria for defining MMSCs [5].

For contact co-culturing with slices of FVB mice brain we used GFPpositive MMSCs of 2-3 passages. Viability of cells for transplantation was $93.6 \%$.

Periventricular leukomalacia modelling on organotypic culture of mice brain slices. Periventricular leukomalacia was modelled by oxygenglucose deprivation (OGD) of the brain sections, followed by addition in culture medium of endotoxin lipopolysaccharide (LPS) to simulate the process of inflammation. OGD was created in a special chamber with an oxygen-free gas medium, which contained $95 \%$ nitrogen (N2) and 5 $\% \mathrm{CO}_{2}$. For OGD, normal culture media was replaced for PBS, $12.5 \mathrm{mM}$ Hepes adding $10 \mathrm{mM}$ D-sucrose instead of glucose. OGD duration was 30 minutes, after which the sections were washed twice and returned to normal culture conditions (normoxic reoxygenation for 24 and 48 hours). After OGD, the culture medium was added with $100 \mathrm{ng} / \mathrm{ml}$ LPS (L4130, Sigma-Aldrich, USA).

MMSCs were applied in suspension directly on cultured brain slices (25.103 cells per one slice) 2 hours before PVL modelling.

In the study we had the following experimental groups: 1 - intact cultured brain slices; 2 - slices after PVL; 3 - intact cultured MMSCs; 4 - cultured MMSCs after PVL; 5 - slices + MMSCs; 6 - slices + MMSCs after PVL.

Quantitative assessment of lactate dehydrogenase (LDH) in the culture medium. Determination of the changes in the relative amount of the cytosolic enzyme LDH in the culture medium was performed by colorimetric method using a test kit G1780 (Promega, USA). While 
damage to the cell membrane LDH is released into the culture medium and characterizes the degree of cell damage. The color intensity is directly proportional to the amount of the enzyme LDG in the culture medium and inversely proportional to the viability of cells in culture [2].

After conducting experiments on organotypic brain sections, we took $200 \mu \mathrm{l}$ culture medium in 24-well plates. In each well we added $200 \mu \mathrm{l}$ of substrate to determine LDH. The samples were incubated at room temperature in the dark for 30 minutes. Then we added $200 \mu$ solution, which stopped the reaction (G1780 kit). Optical density of the samples was measured with a spectrophotometer uniSPEC 2 (LLG, Germany) in a cuvette at wavelength $492 \mathrm{~nm}$. Samples were analyzed at 24 and 48 hours after experimental influences in doubles and determined the average value for each hole. Changes in the relative amount of LDH in the culture medium was expressed in conventional units. Conventional units corresponded the optical density units of the solution, which correlated to the area of tissue in the corresponding hole, and normalized to control.

Immunohistochemical analysis of organotypic culture of brain slices. Identification of transplanted cells and assessment of the damage degree to the nervous tissue caused by PVL, was conducted by immunohistochemistry using primary and secondary antibodies conjugated with fluorescent dyes Alexa Flour 14 days after PVL and application of MMSCs on organotypic culture. Sections of the mice brain were fixed with $4 \%$ solution of formaldehyde in $0.1 \mathrm{M}$ phosphate buffer saline (PBS). Observed sections were blocked in a solution of 0.1 M PBS ( $\mathrm{pH}=7.4$ ) supplemented with $0.5 \%$ bovine serum albumin and $0.3 \%$ Triton X-100. The incubation of the sections in a solution of primary antibodies lasted within 12 hours at $+4{ }^{\circ} \mathrm{C}$. We used the following primary antibodies: anti-GFP (a marker of transplanted cells), dilution 1:7000 (Novus Biologicals, US); anti-NeuN (marker of neuronal nuclei), 1:1000 (Millipore, USA) and anti-Oligodendrocytes (oligodendrocyte marker), 1:1000 (Sigma-Aldrich, USA). Primary antibodies were visualized with corresponding secondary antibodies conjugated with fluorochrome AlexaFluor (Invitrogen, USA). Further the organotypic brain culture slices were covered with medium Immu-MOUNT (Thermo Scientific, USA). Immunohistochemically colored culture was studied using confocal scanning microscope FV1000-BX61WI (Olympus, Japan).

Statistical analysis. Statistical analysis was performed using the software Origin Pro 8.5 (OriginLab Corporation, USA). Sample data included the results obtained from three experiments. The results are shown as the arithmetic mean of the four values $(n=4)$ in each experimental group \pm standard error of the mean (SEM). These were characterized by normal distribution, statistical significance of differences was determined by paired Student t-test, the differences were considered significant at $p<0.05$.

\section{RESULTS AND DISCUSSION}

\section{VIABILITY EVALUATION OF ORGANOTYPIC BRAIN SLICES} AND MIMSCS BY LDG.

In this work we used our previously developed model of PVL in vitro, in which modelling of the brain white matter lesions was achieved by oxygen-glucose deprivation of cultured brain sections and the addition of endotoxin lipopolysaccharide in the culture medium [21].

About $10 \%$ of the GFP-positive cells applied to the cultured cut attached to brain slice (Fig. 1). The rest MMSCs distributed on the surface of a porous membrane.

After 24 and 48 hours from the start of PVL in vitro modeling, the relative amount of $\mathrm{LDH}$ in the culture medium increased compared to control slices 3.1 times and 4.3 times, respectively (Fig. 2). Co-culturing of MMSCs significantly reduced the amount of LDH compared to PVL, both after 24 and after 48 hours (2.4 times and 1.9 times, respectively).

In addition, we investigated the effects of components that provide modelling PVL in vitro, the viability of MMSCs in the culture without
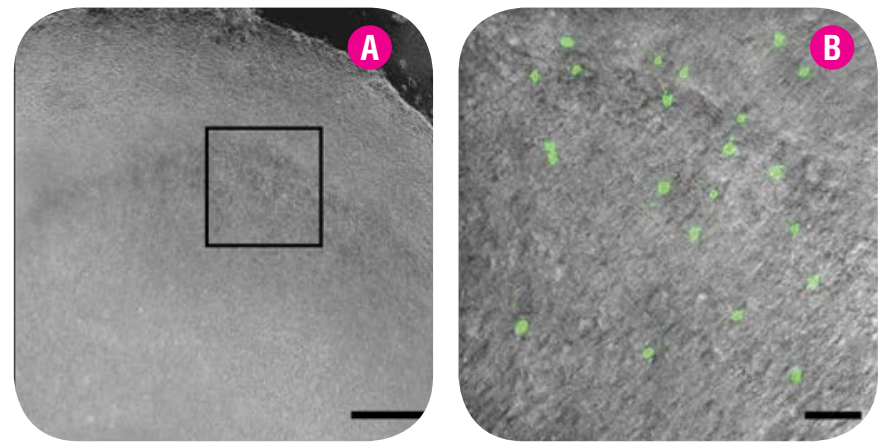

Fig. 1. Microphotographs of the cultured mouse brain slice (A) - area marked with square is increased in Fig. B. GFP-positive MMSCs (green) after application on a cultivated slice. Phase contrast, scale: A - $500 \mu \mathrm{m}, \mathbf{B}-50 \mu \mathrm{m}$.

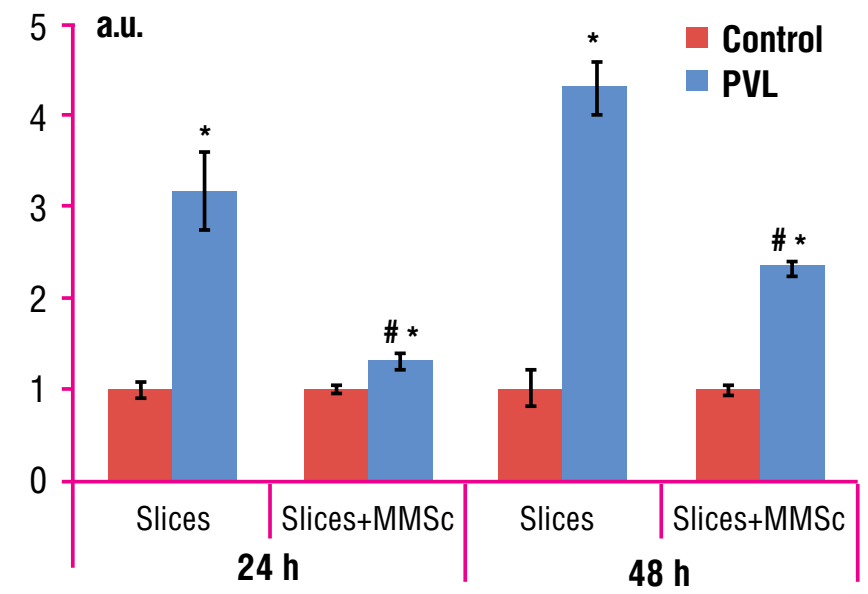

$\Lambda$ of organotypic brain slices 24 and 48 hours after the start of the simulation PVL in vitro (PVL) under culturing slices separately or in co-culture with MMSCs.

Notes: * - statistically significant difference compared to control, \# - statistically significant difference compared to PVL in the respective sections without MMSCs group $(p<0,05)$.

cultured slices. At the $2^{\text {nd }}$ passage in MMSCs culture, fibroblast cells with high adhesiveness, diameter $80 \mu \mathrm{m}$, containing a large number of vacuoles and granules dominated (Fig. 3). 24 and 48 hours after modelling PVL in vitro, relative amount of LDH in MMSCs culture medium did not change significantly compared with control values (without modelling PVL in vitro). That is, the presence of components (damaging factors) that simulate PVL in vitro, did not significantly affect the MMSCs viability in culture, indicating a high resistance of MMSCs to damaging factors used for PVL modelling in this experiment (including 30 min OGD and $100 \mathrm{ng}$ LPS).

Thus, the results of spectrophotometric analysis demonstrated that in vitro modelling of PVL increased the relative amount of LDH in the culture medium compared to control slices, and co-culturing with MMSCs significantly reduced the amount of LDH compared to PVL and had neuroprotective character.

IMIMUNOHISTOCHEMICAL ANALYSIS OF ORGANOTYPIC

CULTURE OF THE BRAIN SLICES.

Immunohistochemical analysis showed that on the 14th day of coculturing of GFP-positive MMSCs with organotypic slices of the brain after PVL a significant number of MMSCs survived and retained the phenotypic 

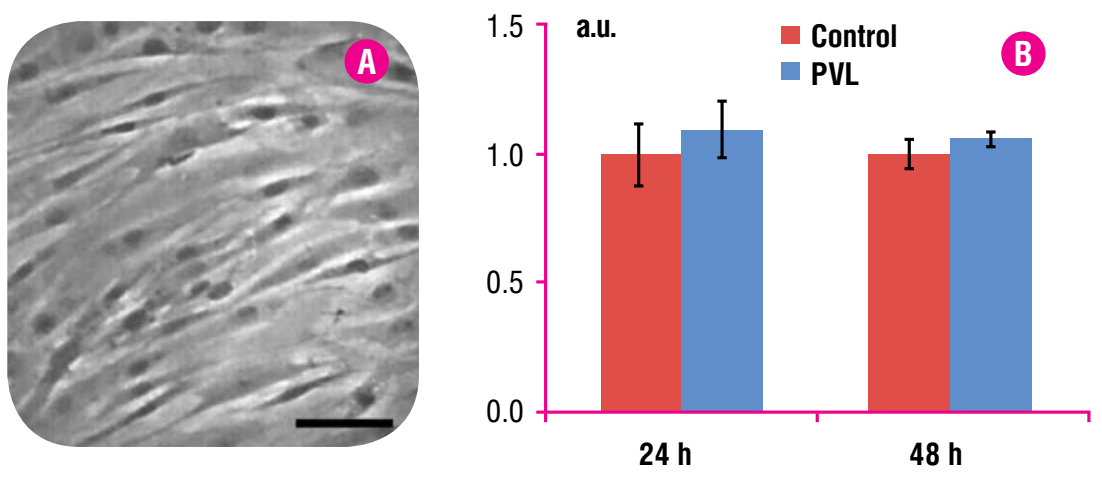

Fig. 3. Impact of PVL in vitro on the viability of MMSCs in culture without cultured slices, 2nd passage. A - microphotographs of MMSCs culture.

B - histogram showing the relative amount of enzyme LDH in culture medium of MMSCs 24 and 48 hours after modelling PVL in vitro. Scale $-300 \mu \mathrm{m}$.
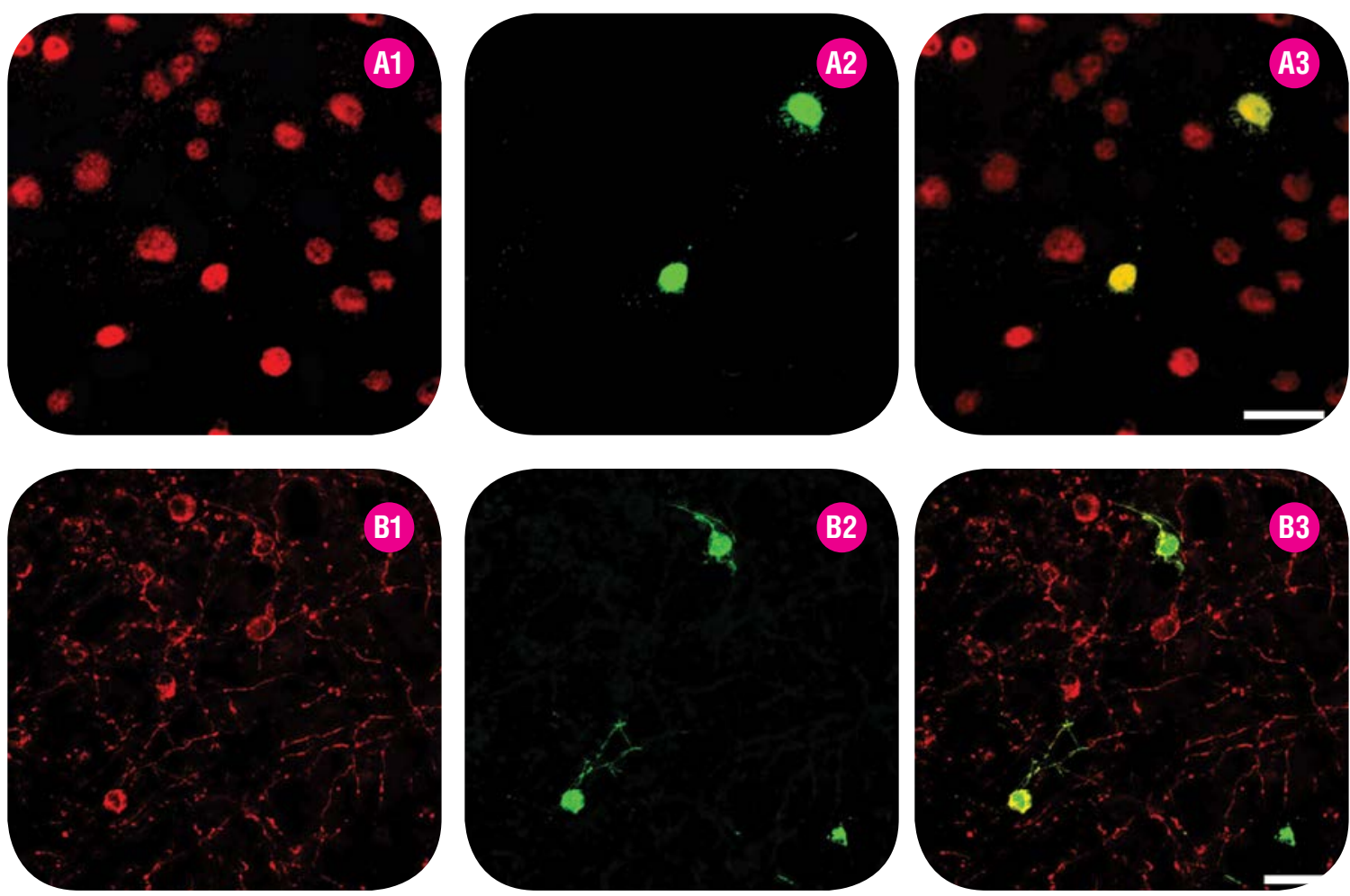

Fig. 4. Immunohistochemical analysis of joint culture of GFP-positive MMSCs and mouse brain slices on the $14^{\text {th }}$ day of co-cultivation. A1 - confocal image of slices stained on neuronal marker NeuN (red color), A2 - GFP-positive MMSCs (green color), $\mathbf{A} 3$ - merge of $\mathbf{A} 1$ and $\mathbf{A} 2$ images (yellow coloring of GFP-positive cells expressing NeuN); B1 - confocal image of slices stained on oligodendrocyte marker 04 (red color), B2 - GFP-positive MMSCs (green color), B3 - merge of B1 and B2 images (yellow coloring of GFP-positive cells expressing oligodendrocytes). Scale - $100 \mu \mathrm{m}$.

traits. Approximately $5 \%$ GFP-positive MMSCs that were adherent to cultured slice, differentiated into mature NeuN-positive neurons (Fig. 4A1A3) or oligodendrocytes (Fig. 4B1-B3).

Thus, immunohistochemical analysis showed that a small number of MMSCs differentiated into neural direction and formed mature neurons and oligodendrocytes on the $14^{\text {th }}$ day of co-culturing of GFP-positive MMSCs with organotypic brain slices after PVL.

Possible mechanisms of MMSCs neuroprotective action can be connected with both the replacement of damaged cells by differentiation and integration of transplanted cells or with bioactive factors that can modulate the development of damage at PVL. It is believed that the MMSCs neuroprotective properties are not mostly implemented directly through differentiation and paracrine due to various factors inducing migration of endogenous neural progenitors in the zone of an injury, stimulate the growth of dendrites and axons and reduce post ischemic inflammation $[13,15,22]$. It was shown that conditioned medium with MMSCs protected neurons culture from induced apoptosis [25]. MMSCs modulate multiple signaling pathways during neurogenesis, angiogenesis, apoptosis and synaptogenesis using transmitters by secretion of fibroblast growth factor (FGF-2), epidermal growth factor (EGF), glial cell derived neurotrophic factor (GDNF) and others. [22]. MMSCs also produce high levels of cytokines, which are involved in the processes of cell proliferation and tissue regeneration, such as IGF-1 (insulin-like growth factor-1), VEGF-a (vascular endothelial growth factor-a), SDF-1 (stromal cell -derived factor-1) and erythropoietin [16].

We know about the influence of MMSCs transplanted on recipient tissue by differentiating into cells specific to the place of transplantation [17]. There is evidence showing that MMSCs of adipose tissue contributed morphological and functional recovery of damaged spinal cord. They expressed glial marker GFAP, $\beta$-tubulin-3 and neural filaments 
NF160 [17]. Our research showed that under PVL at contact co-culturing of MMSCs with brain slices, a certain number of transplanted MMSCs differentiated into neurons and oligodendrocytes as they expressed markers of mature neurons and oligodendrocytes (Fig. 4).

Despite the large amount of data, which indicate a positive effect of MMSCs transplantation in various diseases of the CNS, introduction of stem cell transplantation techniques into clinical practice require additional research and should be based on a thorough understanding of the mechanisms of their functioning and sufficient experimental material. The study of indicated range of questions is possible under experimental transplantation involving adequate models of this disease. Used in vitro model of PVL is adequate to study the mechanisms and means of neuroprotection in this condition.

\section{CONCLUSIONS}

Thus, our results indicate that MMSCs have a protective effect on cultured brain slices and are able to differentiate into neural cells at modelling PVL in vitro.

The study was conducted with the support of the target academic program «Functional genomics and metabolomics in Systems Biology» (registration No. 0112U001475), and the project «Research on the regenerative potential of mesenchymal stem cells in the CNS perinatal pathology" (registration No. 0115U003633).

\section{REFERENCES}

1. Ahn SY, Chang YS, Park WS. Mesenchymal stem cells transplantation for neuroprotection in preterm infants with severe intraventricular hemorrhage. Korean J Pediatr. 2014; 57(6): 251-56

2. Allen M, Millett $P$, Dawes $E$, et al. Lactate dehydrogenase activity as a rapid and sensitive test for the quantification of cell numbers in vitro. Clin Mater. 1994; 16(4): 189-94.

3. Blumenthal I. Periventricular leukomalacia: a review. Eur J Pediatr. 2004; 163(8): 435-42.

4. Chernykh ER, Kafanova MY, Shevela EY, et al. Clinical experience with autologous M2 macrophages in children with severe cerebral palsy. Cell Transplant. 2014; 23(1): 97-104.

5. Dominici M, Le Blanc K, Mueller I, et al. Minimal criteria for defining multipotent mesenchymal stromal cells. The International Society for Cellular Therapy position statement. Cytotherapy. 2006; 8(4): 315-17.

6. Gano D, Andersen SK, Partridge JC, et al. Diminished white matter injury over time in a cohort of premature newborns. J Pediatr. 2015; 166(1): 39-43. doi: 10.1016/j. jpeds.2014.09.009

7. Isik S, Zaim M, Yildiz MT, et al. DNA topoisomerase $\| \beta$ as a molecular switch in neural differentiation of mesenchymal stem cells. Ann Hematol. 2015; 94(2): 7-18.

8. Kan I, Melamed E, Offen D. Autotransplantation of bone marrowderived stem cells as a therapy for neurodegenerative diseases. Handb Exp Pharmacol. 2007; 180: 219-42.

9. Khwaja O, Volpe JJ. Pathogenesis of cerebral white matter injury of prematurity. Arch Dis Child Fetal Neonatal Ed. 2008; 93(2): 153-161. doi: 10.1136/adc.2006.108837

10. Kim JM, Lee ST, Chu K, et al. Systemic transplantation of human adipose stem cells attenuated cerebral inflammation and degeneration in a hemorrhagic stroke model. Brain Res. 2007; 1183: 43-45.

11. Knuesel I, Chicha L, Britschgi M, et al. Maternal immune activation and abnormal brain development across CNS disorders. Nat Rev Neurol. 2014; 10(11): 643-60.

12. Launay E, Gras-Le Guen C, Martinot A, et al. Why children with severe bacterial infection die: a population-based study of determinants and consequences of suboptimal care with a special emphasis on methodological issues. PLoS One. 2014; 9(9): e107286.

13. Lee JA, Kim BI, Jo CH, et al. Mesenchymal stem-cell transplantation for hypoxic-ischemic brain injury in neonatal rat model. Pediatric Research. 2010; 67(1): 42-46.

14. Lindvall O, Kokaia Z. Stem cells in human neurodegenerative disorders - time for clinical translation? J Clin Invest. 2010; 120(1): $29-40$.

15. Linero I, Chaparro O. Paracrine effect of mesenchymal stem cells derived from human adipose tissue in bone regeneration. PLoS One. $2014 ;$ 9(9): 107001.

16. Rasmussen JG, Frøbert $O$, Pilgaard $L$, et al. Prolonged hypoxic culture and trypsinization increase the pro-angiogenic potential of human adipose tissue-derived stem cells. Cytotherapy. 2011; 13(3): 318-28.

17. Ryu HH, Lim JH, Byeon YE, et al. Functional recovery and neural differentiation after transplantation of allogenic adipose-derived stem cells in a canine model of acute spinal cord injury. J Vet Sci. 2009; 10(4): 273-84.

18. Sadan O, Melamed E, Offen D. Bone-marrow-derived mesenchymal stem cell therapy for neurodegenerative diseases. Expert Opin Biol Ther. 2009; 9(12): $1487-497$.

19. Tsai HL, Deng WP, Lai WF, et al. Wnts enhance neurotrophin-induced neuronal differentiation in adult bone-marrow-derived mesenchymal stem cells via canonical and noncanonical signaling pathways. PLoS One. 2014; 9(8): e104937.

20. Tsupykov OM, Kyryk VM, Ustymenko AM, et al. Effect of transplantation of adipose-derived multipotent mesenchymal stromal cells on the nervous tissue and behavioral responses in a mouse model of periventricular leukomalacia. Cell and Organ Transplantology. 2015; 3(1): 68-73.

21. Tsupykov OM, Lushnikova IV, Nikandrova YA, et al. A novel model of periventricular leukomalacia on mouse organotypic brain slice culture. Cell and Organ Transplantology. 2016; 4(2): 188-193. doi:10.22494/cot.v4i2.60

22. Velthoven CTJ, Kavelaars A, Heijnen CJ. Mesenchymal stem cells as a treatment for neonatal ischemic brain damage. Pediatric Research. $2012 ; 71(4)$ : $474-81$.

23. Volpe JJ. Brain injury in premature infants: a complex amalgam of destructive and developmental disturbances. Lancet Neurol. 2009; 8(1): 110-124. doi: 10.1016/ S1474-4422(08)70294-1

24. Wang $L W$, Lin $Y C$, Wang $S T$, et al. Hypoxic/ischemic and infectious events have cumulative effects on the risk of cerebral palsy in very-low-birth-weight preterm infants. Neonatology. 2014; 106(3): 209-215.

25. Adipose stromal cells-secreted neuroprotective media against neuronal apoptosis / X. Wei, L. Zhao, J. Zhong, et al. // Neurosci Lett. - 2009. - Vol. 462, № 1. - P. 76-9.

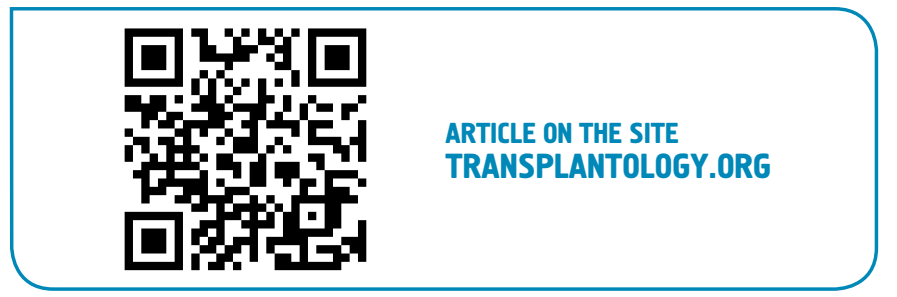

The authors indicate no potential conflicts of interest. 